\title{
Editorial: Psychosomatic Aspects of Obesity
}

\author{
Melissa Ann Kalarchian ${ }^{1 *}$ and Martina De Zwaan² \\ ${ }^{1}$ School of Nursing, Duquesne University, Pittsburgh, PA, United States, ${ }^{2}$ Hannover Medical School, Hanover, Germany
}

Keywords: psychosomatic, bariatric surgery, stigma, cognitive science, renal transplant, obesity

\section{Editorial on the Research Topic}

\section{Psychosomatic Aspects of Obesity}

The prevalence of obesity has increased worldwide to pandemic levels (1). Obesity-related comorbidities can include diabetes, heart disease, and certain types of cancer, as well as depression and eating disorders. Characterized by excess energy intake over expenditure, obesity is a heterogeneous condition with diverse causal and maintaining factors (2). Although there is no cure for obesity, it can be managed with behavioral, pharmacological, and surgical approaches. Given the challenges of treating obesity, there has been increasing emphasis on individualized approaches to prevention and early intervention (3).

This Research Topic includes 7 contributing articles that address various psychosomatic aspects of obesity. Bayer et al. conducted a telephone survey in Germany regarding perceptions of responsibility for maintaining a healthy diet and body weight. Most respondents indicated that responsibility lies with the individual, suggesting a tendency to underestimate the contribution of societal and environmental factors in obesity. This is particularly important given a growing appreciation that public health messages should be based on accurate information about obesity in order to avoid stigma and discrimination (4).

Several articles in this Research Topic touch on obesity and the interplay between impulsivity,

Edited and reviewed by: Stephan Zipfel,

University of Tübingen, Germany

*Correspondence: Melissa Ann Kalarchian kalarchianm@duq.edu

Specialty section: This article was submitted to Psychosomatic Medicine, a section of the journal

Frontiers in Psychiatry

Received: 07 October 2020

Accepted: 22 October 2020

Published: 10 November 2020

Citation:

Kalarchian MA and De Zwaan M (2020) Editorial: Psychosomatic Aspects of Obesity.

Front. Psychiatry 11:614903. doi: 10.3389/fpsyt.2020.614903 inhibition, and reward sensitivity. Naets et al. report on maladaptive eating in children and adolescents with moderate to extreme obesity, evaluating differences in inhibition among 572 participants in a Belgian inpatient treatment center. Results of hierarchical linear regression models suggest that the role of inhibition in weight loss varies by age and gender, highlighting the importance of identifying subgroups for tailoring interventions. Schag et al. also examined differences in inhibition in a pilot study conducted in Germany comparing patients with alcohol use disorder (AUD) to patients with binge eating disorder (BED). Results indicate that patients with AUD and BED process disorder-specific stimuli (alcohol and food, respectively) differently as compared to healthy controls and neutral stimuli. Findings address the ongoing debate surrounding the utility of extending the concept of addiction to the treatment of obesity and eating disorders $(5,6)$.

Schäfer et al. also draw on the field of addiction research, examining decision making with the Cards and Lottery Task, documenting good reliability and validity among individuals in Germany with severe obesity, some of whom were scheduled for bariatric surgery. Lescher et al. also studied decision-making, as measured with a modified version of the Iowa Gambling Task, in bariatric surgery patients as compared to people without obesity in Germany. Food pictures interfered with decisions in both groups, but not in the ways that were predicted. Results of these studies highlight the challenges and opportunities for drawing on the latest findings in cognitive science to develop robust conceptual frameworks, research paradigms, and interventions that can translate to the real world outside of the laboratory setting (7). 
Robitzsch et al. assessed bariatric surgery patients in Germany, considering not only deficits but also psychological resources. Path analyses indicated that depression and eating behavior mediated the relationship between psychological resources and obesity in 127 candidates for surgery. Findings may inform efforts to identify individuals at risk and deliver individualized supports in the service of improving outcomes after bariatric surgery (8).

Finally, it is important to keep in mind that obesity is associated with certain medications and medical conditions. In this Research Topic, Nöhre et al. examined obesity after kidney transplantation among 433 patients in a German posttransplant care program, documenting an association between increasing body mass index and decreasing graft functioning. Rates of post-transplant obesity varied by age and gender. Results again underscore the need for specialized approaches to weight management in vulnerable groups (9).

Collectively, the 7 contributing articles address the psychosomatic aspects of obesity across the lifespan, utilizing a variety of research designs including participants with and without obesity, recruited from community and clinical settings.

\section{REFERENCES}

1. NCD Risk Factor Collaboration (NCD-RisC). Worldwide trends in bodymass index, underweight, overweight, and obesity from 1975 to 2016: a pooled analysis of 2416 population-based measurement studies in 128.9 million children, adolescents, and adults. Lancet. (2017) 390:2627-42. doi: 10.1016/S0140-6736(17)32129-3

2. Blüher M. Obesity: global epidemiology and pathogenesis. Nat Rev Endocrinol. (2019). 15:288-98. doi: 10.1038/s41574-019-0176-8

3. Yanovski SZ, Yanovski JA. Toward precision approaches for the prevention and treatment of obesity. JAMA. (2018) 319:223-4. doi: 10.1001/jama.2017.20051

4. Rubino F, Puhl RM, Cummings DE, Eckel RH, Ryan DH, Mechanick JI, et al. Joint international consensus statement for ending stigma of obesity. Nat Med. (2020) 26:485-97. doi: 10.1038/s41591-020-0803-x

5. Wiss D, Brewerton T. Separating the signal from the noise: how psychiatric diagnoses can help discern food addiction from dietary restraint. Nutrients. (2020) 12:10-2937. doi: 10.3390/nu12102937

6. Brown EC, Park SQ. Obesity and addiction. Nat Hum Behav. (2020) 4:10-1. doi: 10.1038/s41562-019-0622-6

7. Jones A, Hardman CA, Lawrence N, Field M. Cognitive training as a potential treatment for overweight and obesity: a critical review of the evidence. Appetite. (2018) 124:50-67. doi: 10.1016/j.appet.2017.05.032
Although none of the studies reported here are clinical trials, all of them can inform efforts at prevention and intervention that are tailored to the unique needs of individuals who are vulnerable to obesity. This is particularly important as obesity is increasingly recognized as a chronic health condition and a growing public health problem worldwide. Interdisciplinary, multicomponent interventions are needed at the individual, community, and policy levels (10). We hope that this Research Topic will stimulate further work in this important area.

\section{AUTHOR CONTRIBUTIONS}

MK wrote the article. MD critically revised and approved the final version of the manuscript.

\section{ACKNOWLEDGMENTS}

Topic Editors wish to thank all the authors contributing to this Research Topic as well as the reviewers who volunteered their time to provide constructive criticism.

8. Kalarchian MA, Marcus MD. Psychosocial interventions pre and post bariatric surgery. Eur Eat Disord Rev. (2015) 23:457-62. doi: 10.1002/ erv. 2392

9. Sood A, Hakim DN, Hakim NS. Consequences of recipient obesity on postoperative outcomes in a renal transplant: a systematic review and meta-analysis. Exp Clin Transplant. (2016) 14:121-8. doi: 10.6002/ect. 2015.0295

10. Stevens J, Pratt C, Boyington J, Nelson C, Truesdale KP, Ward DS, et al. Multilevel interventions targeting obesity: research recommendations for vulnerable populations. Am J Prev Med. (2017) 52:115-24. doi: 10.1016/j.amepre.2016.09.011

Conflict of Interest: The authors declare that the research was conducted in the absence of any commercial or financial relationships that could be construed as a potential conflict of interest.

Copyright (c) 2020 Kalarchian and De Zwaan. This is an open-access article distributed under the terms of the Creative Commons Attribution License (CC BY). The use, distribution or reproduction in other forums is permitted, provided the original author(s) and the copyright owner(s) are credited and that the original publication in this journal is cited, in accordance with accepted academic practice. No use, distribution or reproduction is permitted which does not comply with these terms. 